Notre Dame Journal of Formal Logic

Volume 48, Number 1, 2007

\title{
Proceedings of the Problem Session
}

\begin{abstract}
Notes are summarized from the problem session held May 19, 2005 at the workshop on Vaught's Conjecture held at the University of Notre Dame. Since the meeting there have been no known efforts which reflect ongoing progress on the problems within these notes.
\end{abstract}

Problem 1 (Young) Suppose $T$ is a complete theory in a fragment. It is possible to have two complete types which cannot be amalgamated. But is it possible to do this if you have only countably many types?

Answer 1 (Baldwin) Yes. See beginning of my survey in BSL [1].

Problem 2 (Buechler) Let $T$ be superstable with less than continuum countable models, and let $p$ be such that $U(p)=\omega$. Show that $\{p(\mathcal{M}): \mathcal{M} \models T$ countable $\}$ contains countably many isomorphism types.

Remark 2 (Buechler) I think this problem is tractable but very hard. Look at work of Newelski on multiplicity. Look at my paper with C. Hoover for an idea of how hard it will be. I think this will be one of the hard parts of $\mathrm{VC}$ for superstable; it will allow an induction.

Problem 3 (Baldwin) Let $\sigma$ be a sentence complete for a fragment of $\mathscr{L}_{\omega_{1} \omega}$ which is $\omega$-stable (reckoned by types in the fragment). Does $\sigma$ satisfy VC?

Remark 3 (Baldwin) This is a problem I thought of a month ago because it would be nice if it were true. Essentially, this asks if we can drop the first-order assumption in the proof of VC for $\omega$-stable theories. Where is compactness needed?

Printed February 26, 2007

(C)2007 University of Notre Dame 
Problem 4 (Sacks) $\quad T$ is scattered. $\mathcal{A} \models T$ is countable. Show that $\mathcal{A}$ has a copy in $L(T)$.

Remark 4 (Sacks) It would not be completely insane to try to do this. We say in $V$ that cardinality of $\mathcal{A}$ is less than $\omega_{1}^{V}$. Is it possible that in $L(T)$ we have $\omega_{1}^{V}$ inaccessible?

\section{Problem 5 (J. F. Knight)}

1. Let $\mathcal{A}$ be a computable structure with noncomputable Scott rank. Then is it true that for any computable infinitary sentence $\sigma$ such that $\mathcal{A} \models \sigma$ there is a computable $\mathscr{B}$ which is not isomorphic to $\mathcal{A}$ but such that $\mathscr{B} \models \sigma$ ?

2. Let $\mathcal{A}, \mathcal{B}$ be two different structures which are both computable with noncomputable Scott rank. Are their index sets $m$-equivalent? (If one is not computably approximable, then its index set would be hyperarithmetical.)

[Note in discussion: Structures as described will not have computable infinitary Scott sentences.]

Problem 6 (Becker) $\quad G$ is big if it has a closed subgroup $H$ with a continuous homomorphism to the group of permutations of $\omega$. Otherwise it is small. It is known that a group satisfies the topological VC iff it is small. Consider the next dichotomy, the Glimm-Effros dichotomy: either you can reduce $E_{0}$ or it reduces to identity on the reals. Do small groups satisfy the Glimm-Effros dichotomy? Groups with a complete left-invariant metric do satisfy this, but there are other possibilities. The automorphism group of Knight's model is small but has no complete left-invariant metric.

[See Knight [3], "A complete $\mathcal{L}_{\omega_{1}, \omega}$-sentence characterizing $\aleph_{1}$," and Hjorth [2], "Knight's model, its automorphism group, and characterizing the uncountable cardinal.”]

Problem 7 (Shami) Assume you have a superstable theory of rank $\omega+1$. Does a positive answer to the analogue to Buechler's problem suffice to prove $\mathrm{VC}$ ?

Problem 8 (Marker) We know that VC holds for o-minimal theories. Is it true for * o-minimal theories, where $*$ is "weakly," "quasi," etc.?

Answer 8 (Baizhanov) For quasi-o-minimal it is known: there are always continuum models.

Problem 9 (Gao) What is the $\mathscr{L}_{\omega_{1} \omega}$ version of VC for Boolean algebras?

Problem 10 (Calvert) Let $K$ be a class of computable structures such that $I(K) \in H Y P$. Is it true that either $E(K) \in H Y P$ or $E(K) \equiv_{m} E$ (Graphs)? 
Problem 11 (Ackerman) For which $\alpha$ are there first-order theories $T$ such that

1. $T$ is scattered $\{\beta: \exists M \models T, \operatorname{sr}(M)=\beta\}=\alpha$,

2. $T$ is weakly scattered,

3. $M \models T \rightarrow \operatorname{sr}(M)=\alpha$.

Problem 12 (Greenberg) Montalban and I looked at classes of structures which are the "well-founded" parts of their superclasses. One of the nice tools for results on these is by coding ordinals inside them. There are also reductions in the other direction, but you need a lot of computation power sometimes to get them, usually to prove that they do what you need. What is the strength of the statement that there is a reduction from superatomic BAs to ordinals? As stated it's third order. Does it prove full arithmetic transfinite recursion? In other words, say $\varphi$ is a second-order arithmetic formula. $\varphi$ defines an "invariant" from (superatomic BAs, well-founded trees, reduced Abelian $p$-groups, compact countable metric spaces, etc.) to ordinals. How much power is needed to do this? It can be done in $\mathrm{ATR}_{0}$. Is this reversible?

Problem 13 (Laskowski) Restrict to $\omega$-stable first-order theories. For which $\omega$ stable $T$ does $\operatorname{Mod}(T)$ have a uniform bound on the Scott height?

Remark 13 (Laskowski) The dividing line is not ENI-DOP. Indeed, there is an ENINDOP $\omega$-stable theory with no bound on the Scott height.

Remark 14 (Baldwin) Let me give one that's more in the spirit of homework.

Problem 14 (Baldwin) Suppose $\sigma \in \mathscr{L}_{\omega_{1} \omega}$ is a counterexample to VC, and let $L$ be the least admissible fragment containing $\sigma$. Show that there is $\mathcal{M} \models \sigma$ which is countable, and some $\mathcal{N}$ such that $\mathcal{M}$ is a proper $L$-elementary submodel of $\mathcal{N}$ but is isomorphic to $\mathcal{N}$. This was already in Gerald Sacks's work. Can you do this without mentioning uncountable models?

\section{References}

[1] Baldwin, J. T., "Notes on quasiminimality and excellence," The Bulletin of Symbolic Logic, vol. 10 (2004), pp. 334-66. Zbl 1064.03023. MR 2083288. 171

[2] Hjorth, G., "Knight's model, its automorphism group, and characterizing the uncountable cardinals," Journal of Mathematical Logic, vol. 2 (2002), pp. 113-44. Zbl 1010.03036. MR 1900550. 172

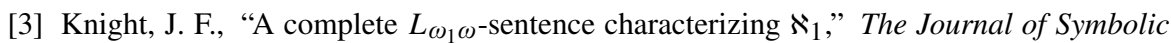
Logic, vol. 42 (1977), pp. 59-62. Zbl 0426.03037. MR 0491141. 172 